\title{
A narrative review of pelvic lymph node dissection in prostate cancer
}

\author{
Douglas C. Cheung ${ }^{1}$, Neil Fleshner ${ }^{1}$, Shomik Sengupta ${ }^{2,3}$, Dixon Woon ${ }^{3}$ \\ ${ }^{1}$ Division of Urology, University of Toronto, Toronto, Canada; ${ }^{2}$ Eastern Health Clinical School, Monash University, Melbourne, Australia; ${ }^{3}$ Urology \\ Unit, Eastern Health, Victoria, Australia \\ Contributions: (I) Conception and design: All authors; (II) Administrative support: None; (III) Provision of study materials or patients: None; (IV) \\ Collection and assembly of data: DC Cheung, D Woon; (V) Data analysis and interpretation: All authors; (VI) Manuscript writing: All authors; (VII) \\ Final approval of manuscript: All authors. \\ Correspondence to: Dr. Dixon Woon. Urology Unit, Eastern Health, Victoria, Australia. Email: dixon.woon@gmail.com.
}

\begin{abstract}
Pelvic lymph node dissection (PLND) is an important component in the staging and prognostication of prostate cancer. We performed a narrative review to assess the literature surrounding PLND: (I) the current guideline recommendations and contemporary utilization, (II) the calculation of patient-specific risk to perform PLND using available nomograms, (III) to review the extent of dissection, and its associated outcomes and complications. Due to the improved lymph node yield, better staging, and theoretical improvement in the control of micro-metastatic disease, guidelines have supported the use of (extended-) PLND in patients deemed to be at intermediate or high risk of lymph node involvement (often at a threshold of $5 \%$ on modern risk nomograms). However, in practice, real-world utilization of PLND varies considerably due to multiple reasons. Conflicting evidence persists with no clear oncological benefit to PLND, and a small, but important, risk of morbidity. Complications are rare, but include lymphoceles; thromboembolic events; and more rarely, obturator nerve, vascular, and ureteric injury. Furthermore, changing disease incidence and stage migration in the context of earlier detection overall have led to a decreased risk of nodal disease. The trade-offs between the benefits, harms, and risk tolerance/threshold must be carefully considered between each patient and their clinician.
\end{abstract}

Keywords: Pelvic lymph node dissection (PLND); pelvic lymphadenectomy; prostate cancer; staging; treatment

Submitted Mar 09, 2020. Accepted for publication Jul 16, 2020.

doi: 10.21037/tau-20-729

View this article at: http://dx.doi.org/10.21037/tau-20-729

\section{Introduction}

Pelvic lymph node dissection (PLND) is an important component in the staging and prognostication of prostate cancer $(1,2)$. Although its utilization was critical in historical series where rates of nodal metastases approached upwards of $25 \%(3,4)$, the introduction of improved screening and the earlier detection of prostate cancer has led to a dramatic stage migration and decline in the incidence of nodal involvement (5). Contemporary series now range from less than $5 \%$ to approximately $10 \%$ depending on the population $(6,7)$. Furthermore, evidence for its therapeutic benefit has been controversial, and may be further reflective of the changing patient population with prostate cancer $(1,8,9)$.

As a result, the benefits, harms, and utilization of PLND have evolved and must be adapted to the modern setting. The aim of this narrative review is to assess the literature surrounding PLND: (I) the current guideline recommendations and contemporary utilization, (II) the calculation of patient-specific risk to perform PLND using available nomograms, (III) to review the extent of dissection, and its associated outcomes and complications.

\section{Indications: guidelines and real-world utilization}

Currently, guidelines recommend PLND in the staging and 
treatment of intermediate to high risk localized patients for the detection of nodal metastases. The European Association of Urology (EAU), European Society for Radiotherapy and Oncology (ESTRO), EAU Section of Urological Research (ESUR), and International Society of Geriatric Oncology (SIOG) guideline (10) recommends an extended pelvic lymph node dissection (e-PLND) in patients with a greater than $5 \%$ risk of nodal involvement (11-13). The American Urological Association (AUA), American Society for Radiation Oncology (ASTRO), and Society of Urologic Oncology (SUO) guideline (14) similarly recommends PLND for all intermediate to high risk patients, although consideration of PLND may be offered to any localized patient. Finally, in the National Comprehensive Cancer Network (NCCN) guideline, e-PLND is recommended at a cut-off of greater than $2 \%$ risk of nodal metastases $(15,16)$.

Despite these recommendations however, large scale studies using administrative datasets across the United States [Surveillance, Epidemiology, and End Results (SEER); and National Cancer Database (NCDB)] indicate that practice patterns and utilization are varied, and may have further room for improvement (17). Nocera et al. reported that $43 \%$ of low risk patients, $75 \%$ of intermediate risk patients, and $88 \%$ of high risk patients receive PLND (18). Similarly, an analysis by Chen et al. found that $79 \%$ of patients with a greater than $5 \%$ risk of nodal involvement (a composite of intermediate and high risk patients) were receiving PLND with radical prostatectomy (19). Taken together, these suggest that utilization may potentially be slightly under-utilized in a small proportion of intermediate and high risk individuals, but equally importantly, potentially over-utilized in a larger proportion of low risk individuals (20). For these low risk individuals in particular, PLND may be unnecessary, comprising avoidable morbidity $(21,22)$ in the context of no clear difference in prostate cancer specific mortality $(1,23)$. This differential utilization in the real world compared to guideline recommendations evidences a clear gap between the identification of those who may benefit from PLND and those who ultimately receive it.

\section{Who gets PLND: calculating patient-specific risk for nodal involvement}

To this aim, numerous nomograms and/or clinical risk algorithms using clinically relevant characteristics have been developed to quantify the risk of nodal involvement and standardize the identification of high risk individuals (24). This was first pioneered with Partin's work to establish prostate cancer staging nomogram tables beginning in the 1990s $(25,26)$. Since then, the updated Briganti and MSK nomograms are currently recommended within the guideline statements for use $(6,15,16,27-29)$. These nomograms demonstrate good discrimination in calibration and validation studies, with area under the curve (AUC) estimates consistently between 0.80 and 0.90 in their cohort data $(27,30,31)$.

In practice, using Gleason grade, clinical stage, PSA, and core involvement in the updated 2017 Briganti nomogram, approximately $1 \%$ of patients with lymph node involvement would be missed while sparing $66 \%$ patients from the morbidity of e-PLND at the predominant guideline threshold of $5 \%$ predicted risk (27). However, despite their robust utility in the traditional setting and across different levels of predicted risk, these nomograms were primarily created in the era preceding routine MRI assessment and cancers detected via targeted biopsy. These contemporary patients, thus, likely have differing risk profiles relative to those identified by traditional biopsy, and question has been raised to the generalizability of these nomograms to predict patient risk following MRI detection (32). Consequently, a recent update to the Briganti nomogram has now been adapted for this setting. For these patients, at the predominant guideline threshold of $5 \%$ predicted risk, approximately $2 \%$ of patients with lymph node involvement would be missed while sparing $51 \%$ patients from the morbidity of e-PLND (7). Finally, it is important to note that different patients may exhibit different levels of risk tolerance relative to the trade-off between PLND positivity and treatment morbidity (and that different nomograms may yield different predictions of risk). In this light, any clinical decision must be made in conjunction with the patient's preferences and their own disease characteristics.

\section{Role for imaging in risk assessment}

In addition to these risk nomograms, modern imaging modalities have also been of interest for their predictive value for lymph node involvement (33). Particularly in the case of patients with a lower predicted risk $(<5 \%)$ using conventional nomograms, imaging has been proposed to play a role in identifying patients who may still benefit from PLND and harbor nodal disease (34).

Here, conventional imaging traditionally has had relatively poor diagnostic accuracy: CT has a sensitivity 
of 0.42 and specificity of 0.82 , and MRI has a sensitivity of 0.39 and specificity of 0.82 , even when assessed against an imperfect gold standard of limited-PLND (1-PLND; where the number of positive reference lesions may be understated) (23,35-37). However, novel contrast agents and molecular imaging have recently upended this paradigm, and have led to substantial interest in MRI- and PET-based imaging for lymph node staging $(14,38)$.

In particular, superparamagnetic iron oxide (SPIO) MRI has greatly improved the detection over conventional MRI, and PSMA-based PET tracers have demonstrated promising results amongst existing ligands $(35,39,40)$. The recent proPSMA study by Hofman et al. demonstrated the superior diagnostic accuracy of PSMA PET-CT scan in high risk patients with an overall sensitivity of $85 \%$ and specificity of $98 \%$ ( $83 \%$ and $99 \%$ in pelvic nodal disease, respectively) (41). Although a direct comparison cannot be drawn, these imaging techniques may yield even greater sensitivity than PLND alone, in addition to the benefits of detecting distant metastases outside of the treatment field. Moreover, when these imaging tests are combined with emerging techniques for sentinel node biopsy and radioguided targeting (e.g., indocyanine green), small scale studies have suggested that almost all patients are correctly identified versus e-PLND management: 100\% sensitivity, $94 \%$ accuracy (42). Taken together and with appropriate patient selection, these may represent less-invasive staging options in the future.

However, at the current time, the majority of the evidence for these remains limited with substantial heterogeneity amongst the diagnostic accuracy and predictive value with these techniques, often ranging by up to an order of magnitude in retrospective and small scale studies $(2,43)$. Furthermore, the clinical significance of nodal deposits detected on novel imaging at time of staging remains to be elucidated, and its ultimate impact, if any, on prognosis is unknown. As a result, these have yet to be formally incorporated into widescale practice and guideline adoption.

\section{Extent of dissection}

Differing limits to the extent of dissection in PLND have been described, ranging from 1-PLND (obturator nodes only) to standard PLND (s-PLND; obturator, and external iliac nodes), e-PLND (obturator; external and internal iliac nodes), and super-extended PLND (se-PLND; obturator; external, internal, and common iliac; pre-sacral; other)
$(1,44)$. Where available, limited randomized comparisons have been reported. However, differing definitions/ terminology are often used interchangeably across the study literature (e.g., substituting e-PLND with se-PLND) or are not documented, and significant heterogeneity limit their direct comparison in outcomes assessment. Additionally, studies are mostly retrospective, and from a single institution/single operator, further limiting their generalizability and must be taken with a grain of salt (1).

\section{Potential oncologic benefits}

To date, randomized data have demonstrated a significant detection benefit for e-PLND/se-PLND over more limited dissections: gross lymph node yield is improved, and detectable lymph node metastases are increased (45-47). In a contemporary cohort from the randomized, phase III trial by Lestingi et al., (s)e-PLND yielded a median of 17 nodes versus 3 from 1-PLND, and detected 6 times more nodal metastases (45). Nevertheless, despite the improved performance on staging and prognostication, no therapeutic benefit was garnered with similar rates of biochemical recurrence and increased complications. These findings are echoed in a recent systematic review of 66 studies by Fossati et al:: no benefit was seen across biochemical recurrence, progression to distant metastases, cancer specific or overall survival with PLND; in a European multi-institutional retrospective study by Preisser et al. demonstrating no difference in oncologic outcomes; and in a large propensitymatched SEER analysis in 2019 by Chen et al. where neither those who received versus those who didn't receive PLND, nor the extent of PLND affected cancer specific or overall survival $(1,19,48)$. Notably, many of these studies were confounded by substantial selection bias (in the case of the cohort studies) or with limited follow-up (in the clinical trials). On the other hand, lymph node yield itself, and thereby extent of PLND, has previously been found to predict the risk of 10-year prostate cancer specific mortality since a landmark historic SEER analysis (49), and at least conceptually, the removal of lymph nodes and potential sources of micro-metastatic disease may lead to theoretical benefit. On balance of these conflicting and controversial data however, convincing evidence of therapeutic benefit with PLND has not been demonstrated.

\section{Risk of complications}

Despite not demonstrating a clear benefit to oncological 
outcomes, morbidity associated with PLND is not uncommon $(8,50)$. The degree of complications are highly variable and correlate to the extent of dissection, remaining a significant consideration when performing PLND $(8,51,52)$. The predominant complications include lymphocele, thromboembolism, obturator injury, ureteric injury, and vascular injury (22).

Lymphoceles are a common complication following PLND, and are positively associated with a greater extent of dissection $(52,53)$. In a contemporary series, Keskin et al. noted that $9 \%$ of patients experienced a lymphocele after e-PLND (54). However, only $2.5 \%$ were symptomatic. This proportion underscores the issue: data series with reflexive post-operative imaging have demonstrated that the post-operative incidence of lymphoceles may be as high as $22 \%$ to $54 \%$, yet only a small proportion will ever become symptomatic and cause morbidity (55-58).

Similarly, thromboembolic events remains a fairly rare occurrence: only a small percentage of these $(0.3-0.5 \%)$ are symptomatic and/or require treatment, although the incidence of subclinical events is higher $(59,60)$. As such, routine pharmacological prophylaxis is not currently recommended in low risk (for thromboembolism) patients with PLND, but may be used for patients at intermediate risk (indicated for e-PLND only) and high risk (indicated for s-PLND and e-PLND) $(61,62)$. For all patients with PLND, mechanical prophylaxis is recommended.

The obturator nerve may be clipped or injured as part of PLND in crush, thermal, and transection injuries $(63,64)$. Although a rare complication, these can lead to significant sensory (medial thigh) and motor (adduction) impairments in the post-operative period, requiring intensive physiotherapy, and if recognized, surgical repair or removal of the offending clip $(65,66)$. In one of the largest consecutive contemporary cohorts of 3,558 laparoscopic and robotic cases, Gozen et al. reported 5 cases $(0.1 \%)$ of obturator nerve injury (67). In 3 cases, clips were removed, and in the other 2 cases, the transected nerve was repaired intra-operatively. Prolonged physiotherapy, pain management, and vitamin B6 were required, but no long-term deficits were noted at followup. Finally, ureteric and vascular injuries $(<1 \%$ each) are extremely rare, but significant complications associated with PLND (50). These are often immediately recognized and repaired at the time of $\mathrm{OR}(8)$.

\section{Conclusions}

PLND is a common and indicated procedure in patients with intermediate to high risk for nodal involvement of their prostate cancer. For these individuals, e-PLND remains the recommended approach due to the improved lymph node yield, better staging, and theoretical improvement in the control of micro-metastatic disease, although the data surrounding the oncological benefits of PLND and its extent remain controversial. Complications are rare for PLND; however, changing disease incidence and stage migration in the context of earlier detection have led to a decreased risk of nodal disease; and the trade-offs between the benefits, harms, and risk tolerance/threshold must be carefully weighed for each patient.

\section{Acknowledgments}

Funding: None.

\section{Footnote}

Provenance and Peer Review: This article was commissioned by the editorial office, Translational Andrology and Urology for the series "Surgery for Urologic Cancers". The article has undergone external peer review.

Conflicts of Interest: All authors have completed the ICMJE uniform disclosure form (available at http:// dx.doi.org/10.21037/tau-20-729). The series "Surgery for Urologic Cancers" was commissioned by the editorial office without any funding or sponsorship. Shomik Sengupta served as the unpaid Guest Editor of the series and serves as an unpaid editorial board member of Translational Andrology and Urology from Jul 2018 to Jun 2020. The authors have no other conflicts of interest to declare.

Ethical Statement: The authors are accountable for all aspects of the work in ensuring that questions related to the accuracy or integrity of any part of the work are appropriately investigated and resolved.

Open Access Statement: This is an Open Access article distributed in accordance with the Creative Commons Attribution-NonCommercial-NoDerivs 4.0 International License (CC BY-NC-ND 4.0), which permits the noncommercial replication and distribution of the article with the strict proviso that no changes or edits are made and the original work is properly cited (including links to both the formal publication through the relevant DOI and the license). See: https://creativecommons.org/licenses/by-nc-nd/4.0/. 


\section{References}

1. Fossati N, Willemse PM, Van den Broeck T, et al. The Benefits and Harms of Different Extents of Lymph Node Dissection During Radical Prostatectomy for Prostate Cancer: A Systematic Review. Eur Urol 2017;72:84-109.

2. Bianchi L, Gandaglia G, Fossati N, et al. Pelvic lymph node dissection in prostate cancer: indications, extent and tailored approaches. Urologia 2017;84:9-19.

3. Gervasi LA, Mata J, Easley JD, et al. Prognostic significance of lymph nodal metastases in prostate cancer. J Urol 1989;142:332-6.

4. Donohue RE, Mani JH, Whitesel JA, et al. Pelvic lymph node dissection. Guide to patient management in clinically locally confined adenocarcinoma of prostate. Urology 1982;20:559-65.

5. Bishoff JT, Reyes A, Thompson IM, et al. Pelvic lymphadenectomy can be omitted in selected patients with carcinoma of the prostate: development of a system of patient selection. Urology 1995;45:270-4.

6. Briganti A, Larcher A, Abdollah F, et al. Updated nomogram predicting lymph node invasion in patients with prostate cancer undergoing extended pelvic lymph node dissection: the essential importance of percentage of positive cores. Eur Urol 2012;61:480-7.

7. Gandaglia G, Ploussard G, Valerio M, et al. A Novel Nomogram to Identify Candidates for Extended Pelvic Lymph Node Dissection Among Patients with Clinically Localized Prostate Cancer Diagnosed with Magnetic Resonance Imaging-targeted and Systematic Biopsies. Eur Urol 2019;75:506-14.

8. Loeb S, Partin AW, Schaeffer EM. Complications of pelvic lymphadenectomy: do the risks outweigh the benefits? Rev Urol 2010;12:20-4.

9. van den Bergh R, Gandaglia G, Tilki D, et al. Trends in Radical Prostatectomy Risk Group Distribution in a European Multicenter Analysis of 28572 Patients: Towards Tailored Treatment. Eur Urol Focus 2019;5:171-8.

10. Mottet N, Bellmunt J, Briers E, et al. EAU - ESTRO ESUR - SIOG Guidelines on Prostate Cancer. Accessed Feb 2020. Available online: https://uroweb.org/wpcontent/uploads/EAU-ESUR-ESTRO-SIOG-Guidelineson-Prostate-Cancer-large-text-V2.pdf

11. Hinev AI, Anakievski D, Kolev NH, et al. Validation of nomograms predicting lymph node involvement in patients with prostate cancer undergoing extended pelvic lymph node dissection. Urol Int 2014;92:300-5.

12. Dell'Oglio P, Abdollah F, Suardi N, et al. External validation of the European association of urology recommendations for pelvic lymph node dissection in patients treated with robot-assisted radical prostatectomy. J Endourol 2014;28:416-23.

13. Abdollah F, Cozzarini C, Suardi N, et al. Indications for pelvic nodal treatment in prostate cancer should change. Validation of the Roach formula in a large extended nodal dissection series. Int J Radiat Oncol Biol Phys 2012;83:624-9.

14. Sanda MG, Cadeddu JA, Kirkby E, et al. Clinically Localized Prostate Cancer: AUA/ASTRO/SUO Guideline. Part I: Risk Stratification, Shared Decision Making, and Care Options. J Urol 2018;199:683-90.

15. Mohler JL, Armstrong AJ, Bahnson RR, et al. Prostate Cancer, Version 1.2016. J Natl Compr Canc Netw 2016;14:19-30.

16. Mohler JL, Antonarakis ES, Armstrong AJ, et al. Prostate Cancer, Version 2.2019, NCCN Clinical Practice Guidelines in Oncology. J Natl Compr Canc Netw 2019;17:479-505.

17. Abdollah F, Abdo A, Sun M, et al. Pelvic lymph node dissection for prostate cancer: adherence and accuracy of the recent guidelines. Int J Urol 2013;20:405-10.

18. Nocera L, Sood A, Dalela D, et al. Rate and Extent of Pelvic Lymph Node Dissection in the US Prostate Cancer Patients Treated With Radical Prostatectomy. Clin Genitourin Cancer 2018;16:e451-67.

19. Chen J, Wang Z, Zhao J, et al. Pelvic lymph node dissection and its extent on survival benefit in prostate cancer patients with a risk of lymph node invasion $>5 \%$ : a propensity score matching analysis from SEER database. Sci Rep 2019;9:17985.

20. Schiffmann J, Larcher A, Sun M, et al. Suboptimal use of pelvic lymph node dissection: Differences in guideline adherence between robot-assisted and open radical prostatectomy. Can Urol Assoc J 2016;10:269-76.

21. Burkhard FC, Schumacher M, Studer UE. The role of lymphadenectomy in prostate cancer. Nat Clin Pract Urol 2005;2:336-42.

22. Briganti A, Chun FK, Salonia A, et al. Complications and other surgical outcomes associated with extended pelvic lymphadenectomy in men with localized prostate cancer. Eur Urol 2006;50:1006-13.

23. Motterle G, Ahmed ME, Andrews JR, et al. The Role of Radical Prostatectomy and Lymph Node Dissection in Clinically Node Positive Patients. Front Oncol 2019;9:1395. 
24. Sengupta S, Weerakoon M, Sethi K, et al. Algorithm for selecting men for pelvic lymph node dissection (PLND) during radical prostatectomy based on clinical risk factors in an Australian population. BJU Int 2012;109 Suppl 3:48-51.

25. Partin AW, Mangold LA, Lamm DM, et al. Contemporary update of prostate cancer staging nomograms (Partin Tables) for the new millennium. Urology 2001;58:843-8.

26. Partin AW, Yoo J, Carter HB, et al. The use of prostate specific antigen, clinical stage and Gleason score to predict pathological stage in men with localized prostate cancer. J Urol 1993;150:110-4.

27. Gandaglia G, Fossati N, Zaffuto E, et al. Development and Internal Validation of a Novel Model to Identify the Candidates for Extended Pelvic Lymph Node Dissection in Prostate Cancer. Eur Urol 2017;72:632-40.

28. Memorial Sloan Kettering Cancer Centre. Dynamic Prostate Cancer Nomogram. Accessed Feb 2020. Available online: https://www.mskcc.org/nomograms/prostate

29. Cimino S, Reale G, Castelli T, et al. Comparison between Briganti, Partin and MSKCC tools in predicting positive lymph nodes in prostate cancer: a systematic review and meta-analysis. Scand J Urol 2017;51:345-50.

30. Hansen J, Rink M, Bianchi M, et al. External validation of the updated Briganti nomogram to predict lymph node invasion in prostate cancer patients undergoing extended lymph node dissection. Prostate 2013;73:211-8.

31. Leyh-Bannurah SR, Budaus L, Pompe R, et al. North American Population-Based Validation of the National Comprehensive Cancer Network Practice Guideline Recommendation of Pelvic Lymphadenectomy in Contemporary Prostate Cancer. Prostate 2017;77:542-8.

32. Dell'Oglio P, Stabile A, Dias BH, et al. Impact of multiparametric MRI and MRI-targeted biopsy on pretherapeutic risk assessment in prostate cancer patients candidate for radical prostatectomy. World J Urol 2019;37:221-34.

33. Watson MJ, George AK, Maruf M, et al. Risk stratification of prostate cancer: integrating multiparametric MRI, nomograms and biomarkers. Future Oncol 2016;12:2417-30.

34. Porpiglia F, Manfredi M, Mele F, et al. Indication to pelvic lymph nodes dissection for prostate cancer: the role of multiparametric magnetic resonance imaging when the risk of lymph nodes invasion according to Briganti updated nomogram is $<5$. Prostate Cancer Prostatic Dis 2018;21:85-91.

35. Sankineni S, Brown AM, Fascelli M, et al. Lymph node staging in prostate cancer. Curr Urol Rep 2015;16:30.
36. Hövels AM, Heesakkers RA, Adang EM, et al. The diagnostic accuracy of CT and MRI in the staging of pelvic lymph nodes in patients with prostate cancer: a metaanalysis. Clin Radiol 2008;63:387-95.

37. Weaver JK, Kim EH, Vetter JM, et al. Prostate Magnetic Resonance Imaging Provides Limited Incremental Value Over the Memorial Sloan Kettering Cancer Center Preradical Prostatectomy Nomogram. Urology 2018;113:119-28.

38. Thoeny HC, Barbieri S, Froehlich JM, et al. Functional and Targeted Lymph Node Imaging in Prostate Cancer: Current Status and Future Challenges. Radiology 2017;285:728-43.

39. Zarzour JG, Galgano S, McConathy J, et al. Lymph node imaging in initial staging of prostate cancer: An overview and update. World J Radiol 2017;9:389-99.

40. Muteganya R, Goldman S, Aoun F, et al. Current Imaging Techniques for Lymph Node Staging in Prostate Cancer: A Review. Front Surg 2018;5:74.

41. Hofman MS, Lawrentschuk N, Francis RJ, et al. Prostatespecific membrane antigen PET-CT in patients with high-risk prostate cancer before curative-intent surgery or radiotherapy (proPSMA): a prospective, randomised, multicentre study. Lancet 2020;395:1208-16.

42. Hinsenveld FJ, Wit EMK, van Leeuwen PJ, et al. ProstateSpecific Membrane Antigen PET/CT Combined with Sentinel Node Biopsy for Primary Lymph Node Staging in Prostate Cancer. J Nucl Med 2020;61:540-5.

43. Woo S, Suh CH, Kim SY, et al. The Diagnostic Performance of MRI for Detection of Lymph Node Metastasis in Bladder and Prostate Cancer: An Updated Systematic Review and Diagnostic Meta-Analysis. AJR Am J Roentgenol 2018;210:W95-109.

44. Mattei A, Fuechsel FG, Bhatta Dhar N, et al. The template of the primary lymphatic landing sites of the prostate should be revisited: results of a multimodality mapping study. Eur Urol 2008;53:118-25.

45. Lestingi JFP, Guglielmetti G, Pontes J Jr, et al. Extended versus limited pelvic lymphadenectomy during radical prostatectomy for intermediate- and high-risk prostate cancer: Early outcomes from a randomized controlled phase III study. J Clin Oncol 2017;35:5018.

46. Schwerfeld-Bohr J, Kämper M, Krege S, et al. Prospective randomized multicenter study comparing limited vs extended pelvic lymphadenectomy in intermediate and high risk prostate cancer. Eur Urol Suppl 2014;13:e270.

47. Clark T, Parekh DJ, Cookson MS, et al. Randomized prospective evaluation of extended versus limited lymph 
node dissection in patients with clinically localized prostate cancer. J Urol 2003;169:145-7; discussion 147-8.

48. Preisser F, van den Bergh RCN, Gandaglia G, et al. Effect of Extended Pelvic Lymph Node Dissection on Oncologic Outcomes in Patients with D'Amico Intermediate and High Risk Prostate Cancer Treated with Radical Prostatectomy: A Multi-Institutional Study. J Urol 2020;203:338-43.

49. Joslyn SA, Konety BR. Impact of extent of lymphadenectomy on survival after radical prostatectomy for prostate cancer. Urology 2006;68:121-5.

50. Musch M, Klevecka V, Roggenbuck U, et al. Complications of pelvic lymphadenectomy in 1,380 patients undergoing radical retropubic prostatectomy between 1993 and 2006. J Urol 2008;179:923-8; discussion 928-9.

51. Zorn KC, Katz MH, Bernstein A, et al. Pelvic lymphadenectomy during robot-assisted radical prostatectomy: Assessing nodal yield, perioperative outcomes, and complications. Urology 2009;74:296-302.

52. Ploussard G, Briganti A, de la Taille A, et al. Pelvic lymph node dissection during robot-assisted radical prostatectomy: efficacy, limitations, and complications-a systematic review of the literature. Eur Urol 2014;65:7-16.

53. Lee HJ, Kane CJ. How to minimize lymphoceles and treat clinically symptomatic lymphoceles after radical prostatectomy. Curr Urol Rep 2014;15:445.

54. Keskin MS, Argun OB, Obek C, et al. The incidence and sequela of lymphocele formation after robotassisted extended pelvic lymph node dissection. BJU Int 2016;118:127-31.

55. Solberg A, Angelsen A, Bergan U, et al. Frequency of lymphoceles after open and laparoscopic pelvic lymph node dissection in patients with prostate cancer. Scand J Urol Nephrol 2003;37:218-21.

56. Spring DB, Schroeder D, Babu S, et al. Ultrasonic evaluation of lymphocele formation after staging lymphadenectomy for prostatic carcinoma. Radiology 1981;141:479-83.

Cite this article as: Cheung DC, Fleshner N, Sengupta S, Woon D. A narrative review of pelvic lymph node dissection in prostate cancer. Transl Androl Urol 2020;9(6):3049-3055. doi: 10.21037/tau-20-729
57. Freid RM, Siegel D, Smith AD, et al. Lymphoceles after laparoscopic pelvic node dissection. Urology 1998;51:131-4.

58. Orvieto MA, Coelho RF, Chauhan S, et al. Incidence of lymphoceles after robot-assisted pelvic lymph node dissection. BJU Int 2011;108:1185-90.

59. Secin FP, Jiborn T, Bjartell AS, et al. Multi-institutional study of symptomatic deep venous thrombosis and pulmonary embolism in prostate cancer patients undergoing laparoscopic or robot-assisted laparoscopic radical prostatectomy. Eur Urol 2008;53:134-45.

60. Clément C, Rossi P, Aissi K, et al. Incidence, risk profile and morphological pattern of lower extremity venous thromboembolism after urological cancer surgery. J Urol 2011;186:2293-7.

61. Thromboprophylaxis in Urological Surgery. Accessed Feb 2020. Available online: https://uroweb.org/wp-content/ uploads/EAU-Guidelines-on-Thromboprophylaxis2018-large-text.pdf

62. Violette PD, Cartwright R, Briel M, et al. Guideline of guidelines: thromboprophylaxis for urological surgery. BJU Int 2016;118:351-8.

63. Fishman JR, Moran ME, Carey RW. Obturator neuropathy after laparoscopic pelvic lymphadenectomy. Urology 1993;42:198-200.

64. Song JH, Kaplan JR, Abbott D, et al. Obturator Compartment Syndrome Secondary to Pelvic Hematoma After Robot-Assisted Laparoscopic Radical Prostatectomy. J Endourol Case Rep 2016;2:141-3.

65. Spaliviero M, Steinberg AP, Kaouk JH, et al. Laparoscopic injury and repair of obturator nerve during radical prostatectomy. Urology 2004;64:1030.

66. Tipton JS. Obturator neuropathy. Curr Rev Musculoskelet Med 2008;1:234-7.

67. Gözen AS, Aktoz T, Akin Y, et al. Is It Possible to Draw a Risk Map for Obturator Nerve Injury During Pelvic Lymph Node Dissection? The Heilbronn Experience and a Review of the Literature. J Laparoendosc Adv Surg Tech A 2015;25:826-32. 